\title{
NEXUS FRAMEWORK FOR WHOLE-CORE FUEL PERFORMANCE: CURRENT APPLICATIONS AND FUTURE TRENDS
}

\author{
Aiden Peakman, Thomas Bennett, Kerr Fitzgerald, Robert Gregg, and Glyn Rossiter \\ National Nuclear Laboratory \\ Chadwick House, Warrington, UK \\ aiden.w.peakman@uknnl.com
}

\begin{abstract}
Current industry practice in fuel licensing often relies on thermo-mechanical modelling of a fuel rod with an artificially constructed bounding power history. The benefit of this approach is that it is computationally efficient; however, the drawbacks are that 1) such an approach is not always conservative, for instance when modelling phenomena related to late onset pellet-clad gap closure; and 2) it can poorly estimate available safety margins for fuel operating at high local power densities and/or to high burnup. For these reasons NNL developed an in-house whole-core fuel performance framework - NEXUS - to enable modelling of all fuel rods in the core using the ENIGMA fuel performance code and computed power histories from core simulation packages (currently limited to PARCS or SIMULATE). One of the main objectives was to create a tool that was both computationally efficient and user friendly. The former was achieved by making use of parallelisable architecture, while the latter was achieved by minimising necessary user input and providing tools for easy interrogation of the fuel performance output.

NEXUS has been applied to several LWR operational scenarios, which we summarise in this paper, including steady-state operation of an ABWR, and a rod ejection accident in a small modular soluble boron free PWR and a GWe-class PWR. We also summarise current development activities related to integrating NNL's in-house fuel performance Monte Carlo uncertainty analysis software CASINO into the NEXUS framework.
\end{abstract}

KEYWORDS: Fuel Performance, ENIGMA, ABWR, Soluble Boron Free, REA

\section{INTRODUCTION}

Historically there has been a reliance in using artificially constructed bounding power histories for fuel performance calculations related to fuel licensing. However, this approach is not always conservative, particularly for fuel phenomena related to late onset of pellet-clad gap closure. To overcome this limitation alternative artificial power histories are often employed (for example cycleaverage power histories, as was the case in licensing of the initial fuel cycles of the Sizewell B reactor) but such an approach can also result in excessive conservatism, for instance when there is a large sensitivity to clad surface temperature and therefore local power density. Furthermore, these conservatisms create challenges for new core designs which are approaching fuel design limits. Alternative approaches that make use of predicted powers for all fuel rods from a validated 
core simulation package are hence becoming increasingly important. For these reasons, NNL has developed a framework called NEXUS that is capable of undertaking whole-core fuel performance in an efficient and user friendly manner.

Within NEXUS modelling of individual fuel rods is based on single runs of the ENIGMA fuel performance code, which are then executed in parallel by NEXUS to enable whole-core fuel performance calculations in a reasonable time-frame (around 10 mins). In ENIGMA, the active stack length of each fuel rod is represented by a series of axial zones, and in each axial zone the fuel is divided into radial annuli of equal thickness. ENIGMA then calculates the thermo-mechanical state of the fuel rod using a finite difference solution scheme as outlined in [1].

NEXUS has been applied to a variety of Light Water Reactor (LWR) cases, including: 1) ABWR fuel performance assessments; and 2) modelling transients in Small PWRs and GWe-class PWRs, which are detailed in this paper. Traditionally, transients modelled in ENIGMA have focused on frequent faults (anticipated operational occurrences) which take place over relatively long timescales; however, work has been undertaken to test the ability of ENIGMA for application to infrequent faults, such as Rod Ejection Accidents (REAs), and to apply this to a whole-core fuel performance assessment via NEXUS as detailed in Section 3.2. Future areas of development of NEXUS to enable greater automation of fuel design and licensing and uncertainty analysis via Monte Carlo methods are also detailed.

\section{ENIGMA VALIDATION AND APPLICATIONS}

ENIGMA is the primary UK computer code for fuel performance calculations in thermal reactors (LWR and Advanced Gas-cooled Reactor systems). ENIGMA is currently capable of modelling fuel behaviour under steady-state and frequent fault transient conditions. Early development of ENIGMA was undertaken as a joint project between NNL (formerly the R\&D division of BNFL) and EDF Energy (formerly CEGB) [1]. However, since 1991 two parallel branches have been developed with NNL's version of ENIGMA concentrating on LWR issues, such as a capability to model MOX and Inert Matrix Fuels. The version of ENIGMA maintained by NNL (ENIGMA-B) is the version detailed in this paper and acts as the fuel performance engine for NEXUS. ENIGMA is available under licence from NNL, or through Studsvik Scandpower. Initial work on NEXUS began with the prototype code APPLES for linking SIMULATE whole-core power history output with ENIGMA [2]. APPLES was superseded by NEXUS and was designed to take advantage of modern HPC architectures. NEXUS was initially developed and tested on a variety commercial PWRs, including modelling an operating PWR in Southern Nuclear's fleet [3].

ENIGMA is validated against a large database of LWR fuel rod irradiations, consisting of over 500 fuel rods irradiated in both commercial and test reactors. The ENIGMA validation database comprises fuel rods up to burnups of $90 \mathrm{GWd} / \mathrm{tHM}$ and encompasses $\mathrm{UO}_{2}$, mixed oxide (MOX), (U, Gd) $\mathrm{O}_{2}$ and zirconia-based inert matrix fuels, with a variety of zirconium-based alloy cladding materials. The validation database comprises mostly PWR but also some BWR fuel rods. ENIGMA has also been applied to modelling thoria-based fuels. ENIGMA has been used for the licensing of $\mathrm{UO}_{2}$ and $(\mathrm{U}, \mathrm{Gd}) \mathrm{O}_{2}$ in the UK's Sizewell B PWR and the Finnish Loviisa VVERs, as well as licensing of MOX fuel in the Swiss Beznau-1 PWR [4]. 


\section{NEXUS Applications}

\subsection{ABWR}

NEXUS has been applied to modelling fuel in an ABWR system using the loading pattern detailed in Ref. [5], with best estimate input parameters and best estimate models. NEXUS analysis focused on studying what are generally the three most limiting fuel performance parameters for $\mathrm{UO}_{2}$ fuelled cores [6]: 1) fuel Rod Internal Pressure (RIP); 2) clad hoop creep strain; and 3) clad oxidation thickness.

It was found that for the core design detailed in Ref. [5] rod internal pressure was the most limiting fuel performance characteristic. The maximum end-of-life rod internal pressure was found to be 7.17 $\mathrm{MPa}$ and occurred in a fuel rod located at the lower edge of the assembly, as shown in Fig. 1. This Fig. 1 was produced via NEXUS using an in-built feature whereby the user can select a parameter and time of interest (in this case end-of-life RIP) and a map of the individual fuel rods is returned by NEXUS, with each rod's unit cell containing a circle with diameter scaled to the value of the parameter of interest at the time of interest.

An end-of-life RIP of 7.17 MPa was at the limit of the conservative design criterion employed, whereby RIP must be less than the coolant pressure $(7.17 \mathrm{MPa})$ to minimise the possibility of pellet clad gap re-opening [7].

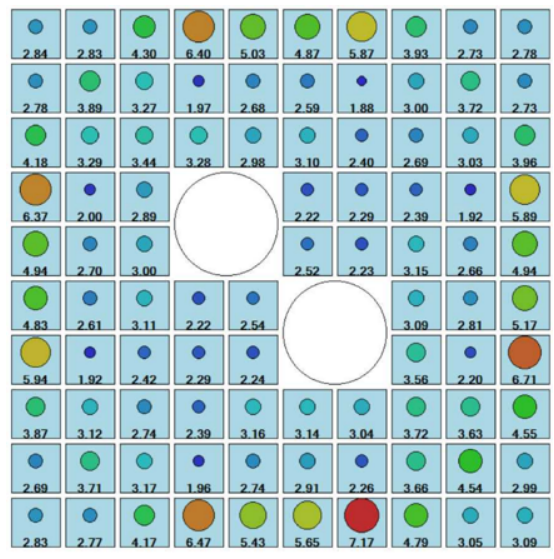

Figure 1: ABWR end-of-life Rod Internal Pressure (RIP) map in units of MPa, within the assembly with the highest RIP at end-of-cycle

Using the results from NEXUS, it is clear (see Fig. 1) that there is scope for the fuel assembly design outlined in [5] to be further improved by allowing for a more heterogeneous radial enrichment within an assembly, in particular, reducing the enrichment of the fuel rods located in the central outer edge of the fuel assembly relative to the other fuel rods. Alternatively, the rod free volume could be increased by making the plenum volume larger by utilising a shorter active fuel height. 


\subsection{Rod Ejection Analysis with PARCS and NEXUS}

As noted in the introduction, NEXUS has been employed to study the behaviour of fuel in a variety of PWR systems, including soluble boron free (SBF) small PWR designs and conventional GWeclass PWRs. Table 1 details the parameters for two PWR configurations that NEXUS has been used to investigate. In this paper, results are presented for modelling Rod Ejection Accidents in the small SBF PWR and GWe-class PWR using CASMO-PARCS-NEXUS.

\section{Table 1: Comparison of core parameters between the small soluble boron free (SBF) PWR} and GWe-class PWR

\begin{tabular}{|c|c|c|}
\hline Parameter & Small SBF PWR & GWe-class PWR \\
\hline Thermal output & $250 \mathrm{MW}$ & $2800 \mathrm{MW}$ \\
\hline Core power density & $69 \mathrm{~kW} / \mathrm{l}$ & $105 \mathrm{~kW} / \mathrm{l}$ \\
\hline Number of fuel assemblies & 52 & 157 \\
\hline Active fuel length & $150 \mathrm{~cm}$ & $366 \mathrm{~cm}$ \\
\hline Lattice configuration & $17 \times 17$ & $17 \times 17$ \\
\hline Inlet temperature & $286^{\circ} \mathrm{C}$ & $286^{\circ} \mathrm{C}$ \\
\hline Outlet temperature & $315^{\circ} \mathrm{C}$ & $322^{\circ} \mathrm{C}$ \\
\hline Cycle length & $13.0 \mathrm{GWd} / \mathrm{tHM}$ & $13.6 \mathrm{GWd} / \mathrm{tHM}$ \\
\hline Number of RCCAs & 52 & 48 \\
\hline
\end{tabular}

The control rod ejection accident is of particular interest, since it is a limiting infrequent fault in PWRs that occurs following the mechanical failure of the control rod drive mechanism. As a result of the high operating pressure in PWR systems, ejection of the control rod typically occurs within a time period of $<1 \mathrm{~s}$. When the reactivity increase is locally greater than $\$ 1$, the core (in the vicinity of the ejected Rod Control Cluster Assembly (RCCA)) is super-critical on prompt neutrons alone. The initial and sudden increase in core power and the subsequent Doppler feedback results in a very short power pulse lasting around $50 \mathrm{~ms}$ [8]. Although short, the reactor power can reach an extremely high value (around several hundred percent of nominal power) depending on the control rod worth of the ejected rod. Fuel rod behaviour is dependent on the power pulse characteristics, as well as coolant and fuel rod conditions (e.g. fuel burnup and initial temperature). During the initial power pulse, the rapidly expanding fuel pellet can result in pellet-clad mechanical interaction (PCMI), potentially leading to cladding rupture. The effect is most limiting when the core is initially in a low power state since the cladding temperature will be relatively low [9].

Fuel vendors have imposed limits on the total amount of energy deposited into the fuel during a transient. Historically, these limits were based on tests on fresh fuel where fuel pin failures were frequently observed above $240 \mathrm{cal} / \mathrm{gUO}_{2}$ [8]. More recent tests on pre-irradiated fuels suggest fuel failure leading to dispersal occurs at much lower energy depositions; hence there is generally 
assumed to be a reducing failure threshold with increasing burnup.

For both PWR designs summarised in Table 1 the rod ejection accident was simulated at beginning of cycle (BOC) from hot zero power (HZP) conditions. For conventional PWRs (i.e. those that use soluble boron for reactivity control in addition to solid burnable poisons and control rods) such a REA is generally most limiting due to fuel conditions and the fact that control rods are typically not inserted deeply into the core during full power operation [8,9]. In contrast, for the small SBF PWR detailed in Table 1 control rod insertion is deepest at start of life and therefore BOC at HZP may not be limiting. Thus, as part of a detailed licensing assessment it would be necessary to consider a range of cases for the small SBF PWR, including the REA initiating from hot full power (HFP) and throughout the cycle to ensure the most limiting case was captured.

For both the small SBF PWR core and GWe-class PWR, the cores were taken to equilibrium in the PARCS models, HZP conditions set and the evolution of a REA computed. Control rod ejection was assumed to take $0.1 \mathrm{~s}$, commensurate with other reactivity initiated accident (RIA) studies [8]. A SCRAM set point of $114 \%$ of nominal core power [8] with a delay of $0.4 \mathrm{~s}$, along with a rod insertion time of 1.0 and $2.0 \mathrm{~s}$ for the small and large cores respectively (noting the shorter active height in the small core) were also assumed. Ultimately, the pin power histories predicted by PARCS were used in a whole-core fuel performance assessment of the transient.

ENIGMA does not include an explicit transient heat transfer model between the clad and coolant, instead relying upon a user supplied heat transfer coefficient. To simplify the method used in this assessment, the steady-state heat transfer coefficient for each pin and axial zone, calculated by ENIGMA at the beginning of the transient, was assumed throughout. This was judged an appropriate approximation for the feasibility assessment presented here given the initial coolant conditions and the adiabatic nature of the initial heat up of the fuel during the initial phases of the transient pulse associated with a REA. A more detailed assessment would need to include a dedicated heat transfer model, either within ENIGMA itself or via further coupling with a subchannel thermal-hydraulics code.

\subsubsection{Small SBF PWR REA Analysis}

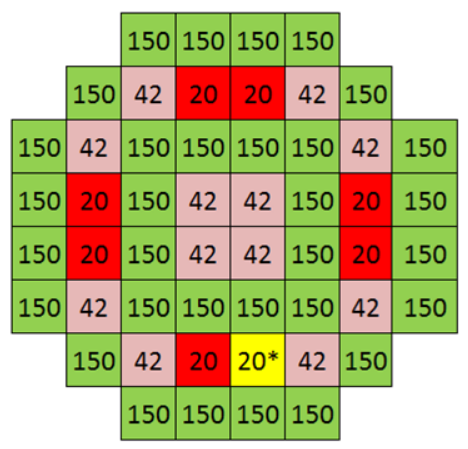

Fully withdrawn (150 steps withdrawn)

Partially inserted bank 1 (42 steps withdrawn)

Partially inserted bank 2 (20 steps withdrawn)

Ejected rod (20 steps withdrawn)

Figure 2: Initial control rod insertion prior to the transient for the small SBF PWR, with the active length of a fuel rod equivalent to 150 steps 
For the small SBF PWR, the reactivity insertion, based on the scenario outlined in Fig. 2, was \$1.24 which resulted in a peak core power of $1114 \%$ of nominal power. The peak fuel pellet-averaged temperature calculated in PARCS was $898 \mathrm{~K}$ (equivalent to a fuel enthalpy of $65 \mathrm{cal} / \mathrm{gUO}_{2}$ ). For comparison, the peak fuel temperature predicted via NEXUS (see Fig. 3) was around $880 \mathrm{~K}$. Comparing Fig. 2 with Fig. 3 it can be seen that whilst rod ejection took place in assembly C7, the peak fuel temperature occurs in assembly B7. The higher fuel temperature in assembly B7 was attributed to this assembly (unlike C7) having a higher fissile isotope concentration, since B7 was freshly loaded at the start of the equilibrium cycle modelled.

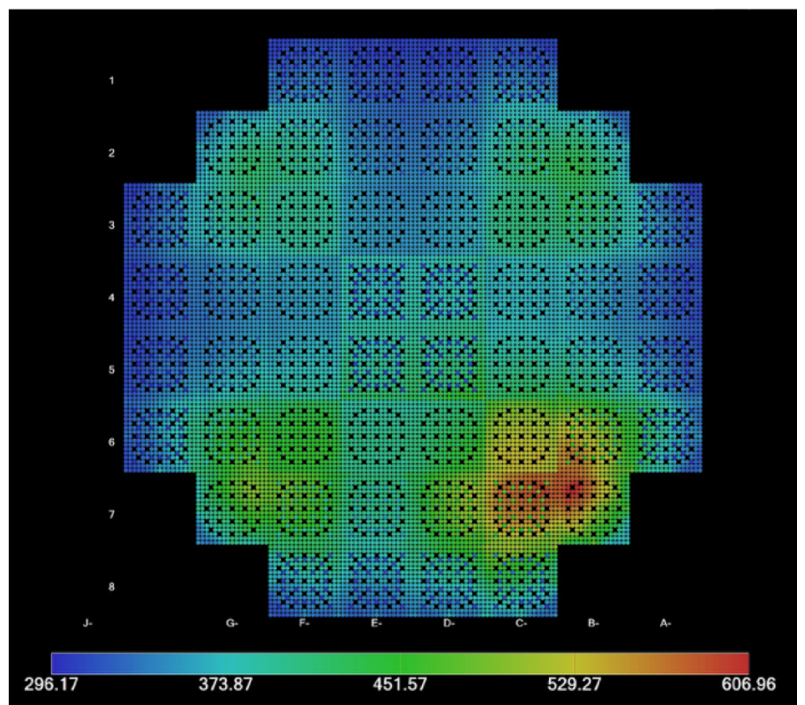

Figure 3: Peak fuel pellet-averaged temperatures $\left({ }^{\circ} \mathrm{C}\right)$ for the small SBF PWR, with assembly $\mathrm{C} 7$ having its RCCA rapidly withdrawn

\subsubsection{GWe-class PWR REA Analysis}

Previous REA studies for conventional large PWRs have considered modification of the crosssections to increase rod worth under REA scenarios [8]. However, for the analysis described here, a hypothetical scenario has been considered where there was less reliance on boron control and higher degree of rod insertion, with several banks being fully inserted, and with the cross-sections unmodified. Even under this hypothetical scenario, reactivity insertion was around $\$ 1.15$, with peak fuel pellet-averaged temperature predicted in NEXUS of around $780 \mathrm{~K}$ (equivalent to a fuel enthalpy of around $55 \mathrm{cal} / \mathrm{gUO}_{2}$ ). The results outlined in this section suggests that the major challenge for the design of a small SBF PWR will be the design of a control pattern which limits the possible reactivity insertion in a control rod ejection scenario.

\section{FUTURE DEVELOPMENTS}

\subsection{CASINO}

To account for model (e.g. fission gas release), power and parameter (e.g. manufacturing tolerances) uncertainties, NNL has developed the CASINO code. CASINO is a Monte Carlo 'wrapper' 
code, intended to facilitate and largely automate the carrying out of sensitivity analyses and uncertainty analyses using ENIGMA, and to provide statistical analysis of the results obtained [4]. In addition, CASINO is parallelised to allow individual Monte Carlo runs to be executed at the same time. By using CASINO's capability to apply uncertainty factors to whole-core power histories from a neutronics package, this avoids the need to use bounding power histories.

NEXUS is currently able to create the necessary base-case ENIGMA fuel rod parameter input for individual pin types, which can then be combined with pin power history data to perform CASINO runs. Furthermore, developments within NEXUS are planned to allow NEXUS to automatically output complete ENIGMA input files (fuel rod parameter and power history input) for fuel pins that are most limiting with respect to certain design criteria (e.g. those on rod internal pressure or clad oxide thickness) to allow easy assessment for rods most pertinent for uncertainty analysis.

\subsection{Automated fuel rod design assessments}

NEXUS already includes a restart capability for performing branching calculations at a particular point during cycle length (e.g. beginning, middle or end of cycle). Therefore, with an automated route to model relevant transients in conjunction with the core simulator packages (e.g. SIMULATE) the necessary best estimate fuel licensing assessments can be carried out. In addition to this capability, the ongoing work to incorporate Monte Carlo uncertainty analysis into NEXUS and using the best estimate runs to determine the most limiting rods for criteria of interest (e.g. rod internal pressure), should enable automated best estimate plus uncertainty fuel rod design assessments. Hence, proposed loading patterns which meet the core design limits (e.g. on power peaking) but not on one or more fuel rod design limits (e.g. on clad hoop creep strain) could be identified at an early stage, saving much time and effort in the loading pattern development process. Furthermore, a pre-cycle assessment could be used to determine the fuel performance implications of any planned operational manoeuvres during the cycle.

\subsection{Fuel performance analysis of infrequent faults}

Work is underway to extend and validate ENIGMA for LOCA simulation via the development of high temperature clad creep and clad oxidation models (including the effects of any alpha to beta phase change), with NEXUS whole-core runs performed prior to the LOCA to predict the initial state of the fuel rods before the LOCA event is simulated. Furthermore, the basic RIA capability tested (see Section 3.2) will be enhanced and validated. The predictions from ENIGMA can then be used with suitable fuel failure criteria to determine whether a given LWR rod will fail. This information, when applied to all fuel rods in the core via NEXUS, will allow the determination of a whole-core fuel failure census in LOCA or potentially RIA assessments; however, most regulatory regimes only apply a limit on the number of rods that fail (e.g. 10\%) for LOCA assessments. The results from such a census calculation can in turn be used for accurate determination of source terms for reactor licensing.

\section{CONCLUSIONS}

The reduced barriers to computational power have enabled whole-core fuel performance to be readily applied to fuel design assessments, which helps reduce issues related to the standard industrial 
route where a small number of artificially constructed power histories are used in fuel performance assessment. NNL's whole-core fuel performance framework - NEXUS - has been applied to a variety of LWR assessments, with three examples presented in this paper: ABWR steady-state analysis; the simulation of a REA in small SBF PWR; and a REA assessment in a conventional GWe-class PWR.

Whilst most NEXUS assessments have focused on using best estimate models with best estimate input parameters, recent developments to parallelise NNL's CASINO Monte Carlo sensitivity and uncertainty analysis code is further adding to capabilities within NEXUS. This additional capability is allowing for greater automation in fuel design assessments. Finally, developments underway in ENIGMA for LOCA and RIA simulation is allowing for the use of NEXUS to undertake wholecore fuel failure census calculations.

\section{ACKNOWLEDGEMENTS}

CASINO and NEXUS development were funded through NNL's Internal R\&D programme. The NEXUS applications presented here were funded by the UK government's Department for Business, Energy and Industrial Strategy, as part of the Nuclear Innovation Programme (Advanced Fuels - TRN 1210/09/2016(1)).

\section{REFERENCES}

[1] G. Rossiter. "Development of the ENIGMA Fuel Performance Code for Whole Core Analysis and Dry Storage Assessments." Nuclear Engineering and Technology, volume 43, pp. 489498 (2011).

[2] R. Gregg. "Whole core fuel performance calculations." In IAEA TCM on water reactor fuel performance modelling. Kendal (2005).

[3] A. Alapour, R. Joyce, A. DiGiovine, S. Tarves, A. Worrall, and R. Gregg. "Robust PCI monitoring during PWR operation at Southern Nuclear." In ANS International. Orlando (2010).

[4] G. Rossiter. "Development of the ENIGMA fuel performance code." In Studsvik International Core Management Software Users' Group Meeting. Madrid (2013).

[5] A. Peakman, C. Grove, K. Fitzgerald, and R. Gregg. "Development of an equilibrium loading pattern and whole-core fuel performance assessment in the Advanced Boiling Water Reactor with UO2 and U3Si2 fuels." Progress in Nuclear Energy, volume 117, p. 103053 (2019).

[6] A. Peakman, H. Owen, and T. Abram. "The core design of a Small Modular Pressurised Water Reactor for commercial marine propulsion." Progress in Nuclear Energy, volume 113, pp. 175 - 185 (2019).

[7] D. D. Baron and L. Hallstadius. "Fuel Performance of Light Water Reactors." In Comprehensive Nuclear Materials, chapter 2.19, pp. 481-514. Waltham (2012).

[8] D. Diamond, B. Bromley, and A. Aronson. Studies of the Rod Ejection Accident in a PWR. NRC, New York, USA (2002).

[9] OECD-NEA. Nuclear Fuel Behaviour Under Reactivity-initiated Accident (RIA) Conditions. OECD-NEA, Paris, France (2010). 